\title{
Correction to: Reasons for lithium discontinuation in men and women with bipolar disorder: a retrospective cohort study
}

Louise Öhlund ${ }^{1}$, Michael Ott ${ }^{2}$, Sofia Oja ${ }^{3}$, Malin Bergqvist ${ }^{4}$, Robert Lundqvist ${ }^{5}$, Mikael Sandlund ${ }^{6}$, Ellinor Salander Renberg ${ }^{6}$ and Ursula Werneke $e^{1,7^{*}}$

\section{Correction}

Following publication of the original article [1], the authors reported an error in Fig. 1 and Table 1, concerning the number of female participants. The correct number is 283 , instead of 238 that was originally published.

\section{Author details}

${ }^{1}$ Sunderby Research Unit, Department of Clinical Sciences, Division of Psychiatry, Umeå University, Umeå, Sweden. ${ }^{2}$ Department of Public Health and Clinical Medicine, Division of Medicine, Umeå University, Umeå, Sweden. ${ }^{3}$ Department of Psychiatry, Sunderby Hospital, Luleå, Sweden. ${ }^{4}$ Department of Psychiatry, Piteå Älvdals Hospital, Piteå, Sweden. ${ }^{5}$ Research and Innovation Unit, Luleå, Norrbotten Region, Sweden. ${ }^{6}$ Department of Clinical Science,

Division of Psychiatry, Umeå University, Umeå, Sweden. 'Sunderby Hospital Psychiatry, 97180 Luleå, Sweden.

Received: 18 September 2018 Accepted: 18 September 2018

Published online: 03 October 2018

\section{Reference}

1. Öhlund L et al. (2018) Reasons for lithium discontinuation in men and women with bipolar disorder: a retrospective cohort study. BMC psychiatry 18:37. https://doi.org/10.1186/s12888-018-1622-1.

\footnotetext{
* Correspondence: uwerneke@gmail.com

${ }^{1}$ Sunderby Research Unit, Department of Clinical Sciences, Division of Psychiatry, Umeå University, Umeå, Sweden

${ }^{7}$ Sunderby Hospital - Psychiatry, 97180 Luleå, Sweden
} 